\title{
Analysis of 3D-EBSD Datasets Obtained by FIB Tomography
}

\author{
P.J. Konijnenberg, A. Khorashadizadeh, S. Zaefferer and D. Raabe \\ Max-Planck-Institute for Iron Research, Düsseldorf Germany
}

3D-EBSD represents a destructive and crystallographic tomographic method. It comprises a repeated sectioning and subsequent surface characterization by Electron Backscatter Diffraction (EBSD). Several sectioning alternatives exist, most widespread though, is an integrated in-situ sectioning technique in which an SEM based focused ion beam (FIB) is applied to generate accurately spaced serial sections.

In contrast to scalar imaging techniques, in EBSD each pixel or volume pixel (voxel) contains a high dimensional information vector. On top of that, compared to 2D-EBSD, datasets acquired by 3D-EBSD scale by an additional dimension. Typical dataset sizes currently range in the order of magnitude of $10^{7}$ voxels. All this imposes not only practical limitations on the acquisition conditions, but also sets demands to the post-processing architecture.

EBSD scans tend to suffer from a more or less pronounced drift; a discrepancy between the assumed and the real position of the source point of the Kikuchi patterns. In general, with increased acquisition time spans, as they are typical for 3D-EBSD, capacitive drift phenomena also tend to become more pronounced. The spatial extension of drift also tends to be spatially anisotropic; more pronounced in the direction of the slowest running acquisition direction as compared to the fastest running acquisition direction.

Voxel datasets, as generated by 3D-EBSD, are very useful for a number of basic characterizations, which are less accessible or simply incomplete by 2D-EBSD. This ranges from distributions, such as 3D-grain sizes and shapes, volume fractions to more advanced characterizations such as the full geometrically necessary dislocation tensor.

The key advantage, however, of 3D-EBSD is the option to analyse grain- and phase boundaries in 3 dimensions. Specifically chosen boundaries or boundary sets can be analyzed in a localized, nonstatistical way, resulting in a near complete characterization by misorientation and boundary inclination. A 5-parameter analysis not only yields more precise coordinates of a boundary in boundary space, it allows also for a much more detailed crystallographic understanding.

Common to all EBSD techniques, and with that also to 3D-EBSD, is that they produce discrete representations of the probed volume or area. In consequence, prior to any 5 parametric boundary analysis, semi-continuous boundaries need to be reconstructed from a discrete voxel representation, fig. 1 .

A boundary reconstruction comprises two steps; identifying the boundary surface and subsequently translating this into a mesh of discrete geometric primitives. In the current work a so-called Marching Tetrahedra algorithm is applied, by which the boundary structure is described in terms of boundary primitives, triple and quadruple junctions.

The as-meshed boundary structure, however, still exhibits an undesirable degree of inherent roughness. Our smoothing strategy is physically based, and applies a simulated grain growth, albeit to a very limited extent. 
In spite of all smoothing, persistent artifacts of experimental and algorithmic origin need to be addressed. When boundary normals are mapped onto a typically much smaller (asymmetric) crystal domain, their relative error is enlarged accordingly. This underlines the importance of the accuracy of the boundary reconstruction process, fig. 2 .

In our contribution we report about recent developments in the aforementioned issues, within frames of an in-house post-processing software project, QUBE. We will demonstrate the calculation and representation of geometrically necessary dislocation densities, strategies to reconstruct accurate interfaces from voxel representations, as well as possibilities and limitations of a 5-parameter boundary analysis, fig. 2 .

[1] Konijnenberg P.J et al, Materials Science Forum 702-703 (2011) p. 475-478.
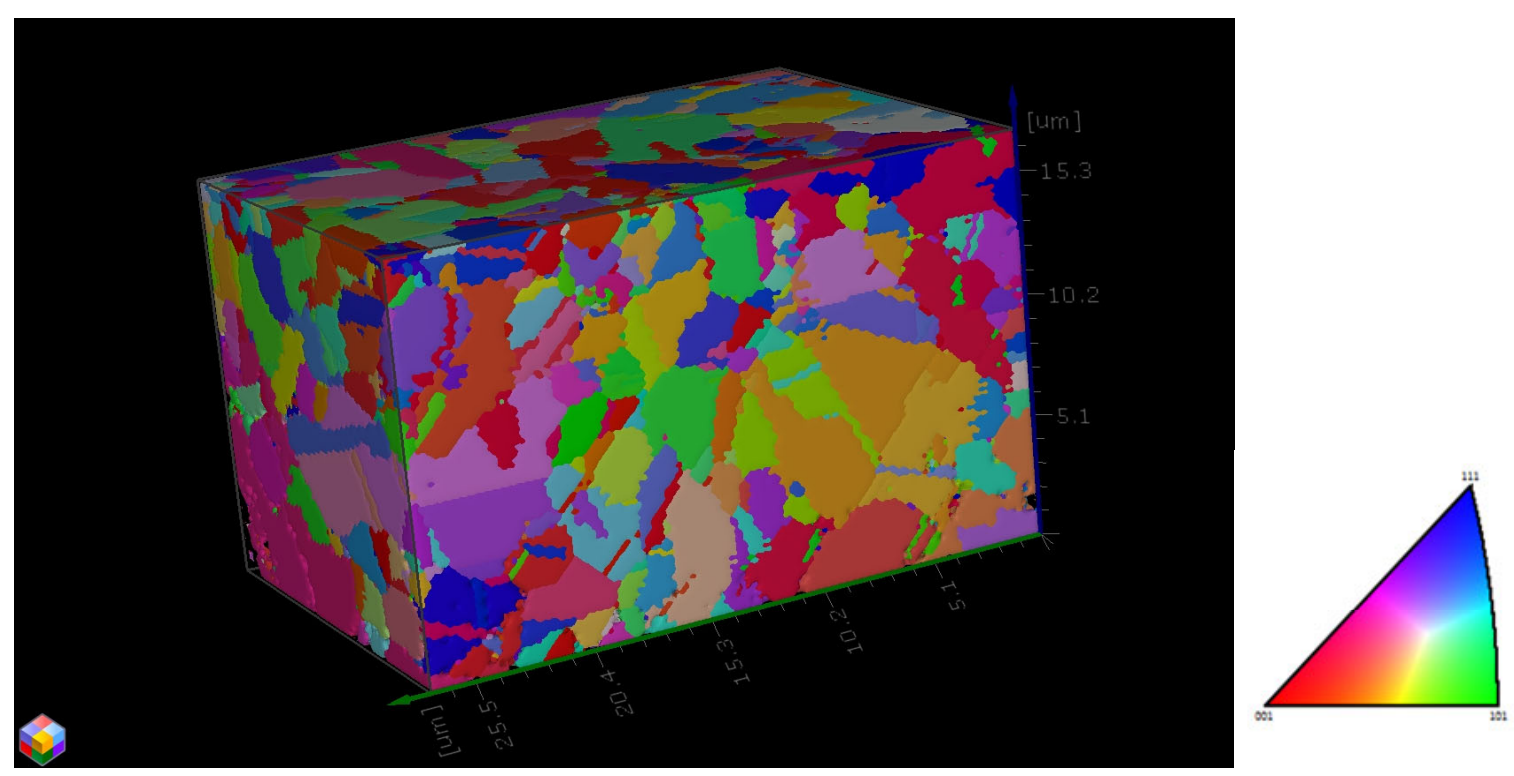

Figure 1. $\mathrm{Cu} 0.17 \mathrm{wt} \% \mathrm{Zr}$, interface dataset, grain orientation color coded (IPF).
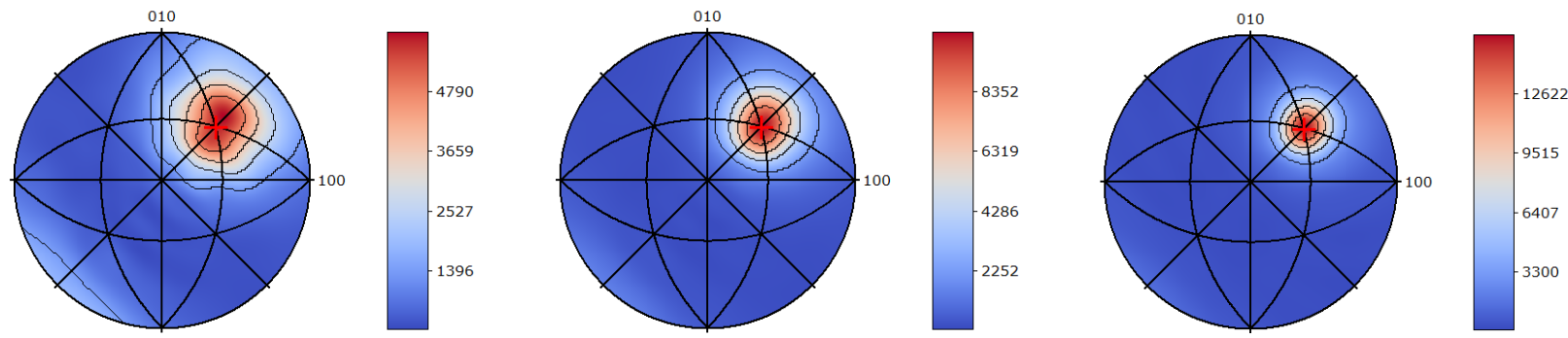

Figure 2. Boundary normal distribution of a twin boundary subset $\left(60^{\circ}<111>, \pm 5^{\circ}\right)$ from the dataset in fig.1, increasing number of smoothing cycles from left to right. 